\title{
Interpretation of negative central shear electron internal transport barriers
}

\author{
André L Rogister \\ Institut für Plasmaphysik, Forschungszentrum Jülich GmbH, Trilateral Euregio Cluster, \\ EURATOM Association, D-52425 Jülich, Germany \\ E-mail: a.rogister@fz-juelich.de
}

Received 27 October 2005, in final form 13 December 2005

Published 20 April 2006

Online at stacks.iop.org/PPCF/48/A341

\begin{abstract}
Box-type electron temperature profiles observed in negative central shear plasmas with electron internal transport barriers are explained with the help of a novel instability mechanism that is predicted when keeping account of the radial component of the trapped electron $\mathrm{gradB}$ and curvature drifts in the theory of the trapped electron mode. These radial velocity components lead to a new term in the quasi-slab radial eigenvalue equation which modifies the asymptotic behaviour in such a way that growing-decaying pairs of bounded solutions are obtained (instead of the usual magnetic shear damped solutions) if $\hat{s} L_{\mathrm{Te}}>0$. There are no bounded solutions if $\hat{s} L_{\mathrm{Te}}<0$. As $L_{\mathrm{Te}}=T_{\mathrm{e}} / \partial_{r} T_{\mathrm{e}}$ is usually negative, instability requires negative magnetic shear $\hat{s}$. The driving mechanism is largest for quasi-slab modes in view of the poloidal angle dependence of the radial drift components. The ratio $Q_{\mathrm{e}} / T_{\mathrm{e}} \Gamma_{\mathrm{e}} \sim\left(q R \omega_{\mathrm{e}, l}^{*} / c_{\mathrm{s}}\right)^{2}$, where $Q_{\mathrm{e}}$ is the anomalous electron energy flux and $\Gamma_{\mathrm{e}}$ the particle flux, is quite large owing to the non-adiabatic trapped electron response and the condition $\left(q R \omega_{\mathrm{e}, l}^{*} / c_{\mathrm{s}}\right)^{2} \gg 1$ that must be satisfied to avoid ion Landau damping on the side-bands $\left(\omega_{\mathrm{e}, l}^{*}\right.$ is the electron diamagnetic frequency, $l$ the toroidal mode number, $1 / q R$ the parallel wave vector of the side-bands and $c_{\mathrm{s}}=\sqrt{T_{\mathrm{e}} / m_{\mathrm{i}}}$ the sound speed).
\end{abstract}

\section{Introduction}

Internal transport barriers (ITBs) are often observed in reversed magnetic shear (RS) plasmas. In most cases, these are produced by additional heating during a fast inductive current ramp-up phase, in order to hold up penetration, and maintained by auxiliary current drive schemes and the self-generated bootstrap current; the inductive current component however remains dominant as, for example, in JT-60U [1]. In the TCV tokamak [2], in contrast, fully non-inductive RS plasmas have been obtained by switching off and replacing the inductive current and Ohmic heating with near-axis electron cyclotron current drive and electron 
cyclotron heating $(\mathrm{ECCD} / \mathrm{ECH})$; magnetic shear is then controlled by perturbations of the inductive current. In the JT-60U experiment, the region of large electron temperature gradient corresponds to weak (positive as well as negative) magnetic shear $(\hat{s})$; inside the barrier, a flat electron temperature profile is associated with large negative values of $\hat{s}$; finally, outside the barrier, a moderate temperature gradient corresponds to positive $\hat{s}$. When near-axis ECH / ECCD is applied to such a plasma with an ITB pre-established by lower hybrid current drive (LHCD) and neutral beam injection, $T_{\mathrm{e}}$ increases mostly inside the barrier whose position remains unchanged. There is no clear region with a particularly sharp density gradient. Qualitatively similar conclusions are reached on the TCV tokamak.

The flatness of the electron temperature profile in the very region where the $\mathrm{ECH}$ power is applied reveals strong anomalous electron heat transport. It has been argued on the basis of existing theories that the plasma is stable in that region and, therefore, that the transport anomaly arises in connection with 'turbulence spreading' [3]. In this paper, we show that theory actually predicts a negative magnetic shear collisionless instability when the full trapped electron response is taken into account; the frequency $\omega^{\prime}$ of the unstable modes in the $E \times B$ rotating frame being the electron diamagnetic frequency $\omega_{\mathrm{e}}^{*}$, they are identified as trapped electron modes (TEMs). Destabilization arises here through the bounce average of the radial component $(\propto \sin \theta, \theta$ being the poloidal angle measured anti-clockwise from the outboard mid-plane) of the bulk trapped electrons vertical drift, $v_{B \text {,trap }}$ (which is the sum of the curvature and $\nabla B$ drifts), weighted by the non-adiabatic distribution function. The growth rate is therefore expected to be small for ballooning modes, motivating us to concentrate on the quasi-slab branch. The above negative magnetic shear instability is to be contrasted with the resonant instability that is triggered by those trapped electrons whose poloidal component $(\propto \cos \theta)$ of $v_{B \text {,trap }}$ (which is a function of the electron velocity) matches the wave poloidal phase velocity [4]. The latter occurs for $0.64 \hat{s}-0.57>0$ and is relevant to the region outside the electron ITB [5].

We have found that side-bands (s-b) play an essential role in quasi-slab approximation as they increase

(i) the effective parallel wave vector $\left[\left(k_{\|}\right)_{\mathrm{s}-\mathrm{b}}=1 / q R\right]$ and, therefore, ion Landau damping (if $\eta_{\mathrm{i}}=\partial_{r} \ln T_{\mathrm{i}} / \partial_{r} \ln N_{\mathrm{i}}>0$ ) [6,7], thereby limiting the unstable spectrum;

(ii) the characteristic radial length of the mode by the neoclassical factor $\sqrt[4]{1+2 q^{2}}[6,7]$ and

(iii) the anomalous electron heat flux $Q_{\mathrm{e}}$ as well as the ratio $Q_{\mathrm{e}} / T_{\mathrm{e}} \Gamma_{\mathrm{e}, \mathrm{i}}$ (where $\Gamma_{\mathrm{e}}$ is the anomalous particle flux) by the factor $\left(q R \omega_{\mathrm{e}, l}^{*} / c_{\mathrm{s}}\right)^{2}$; the latter must be larger than unity for negligible ion Landau damping $\left(c_{\mathrm{s}}=\sqrt{T_{\mathrm{e}} / m_{\mathrm{i}}}\right.$ is the sound speed) [7].

The latter result and the behaviour of the collisionless trapped electron growth rate for negative as well as positive magnetic shear offer an attractive explanation of the complete electron temperature profile and the difference from the density profile. (Definitive conclusions would, of course, require calculation of the saturated turbulence spectrum, leading to the anomalous transport level, and of the particle and energy sources.)

The paper is organized as follows. The major new theoretical results concerning both linear stability theory and the quasi-linear fluxes are presented in section 2. Interpretation of electron ITBs is the subject of section 3. A summary and further remarks are given in section 4 .

\section{Trapped electron instability, revisited}

The radial $(\propto \sin \theta)$ and poloidal $(\propto \cos \theta)$ projections of the ion $\nabla B$ and curvature drifts couple the poloidal Fourier components $m$ and $m \pm 1$ corresponding to a given toroidal mode number $l ; m=-l q\left(r_{l, \mathrm{~m}}\right)$ since the modes are centred on rational surfaces. This 
coupling may (1) generate side-bands and (2) arise from the overlapping of modes centred on neighbouring rational surfaces. We showed in section 6 of [7] that the solutions of the TEM eigenvalue equation obtained when postulating that the term which describes the latter coupling process is dominant are inconsistent with the prerequisite that the characteristic radial length is larger than the distance between neighbouring rational surfaces: coupling due to overlapping, therefore, is never dominant and the 'quasi-slab' ion response applies in all cases. Since the novel instability mechanism considered here for negative magnetic shear is proportional to $\int_{-\theta_{\mathrm{b}}}^{\theta_{\mathrm{b}}} \sin \theta \hat{g}_{\mathrm{e}, l}\left(k_{\mathrm{r}} \Delta_{l}-\theta\right) \mathrm{d} \theta / \sqrt{\cos \theta-\cos \theta_{\mathrm{b}}}$, where $\hat{g}_{\mathrm{e}, l}$ is the non-adiabatic trapped electron response, $\theta_{\mathrm{b}}$ the bouncing angle, $k_{\mathrm{r}}$ the radial wave number and $\Delta_{l}=-1 / l \partial_{\mathrm{r}} q$ the distance between neighbouring rational surfaces, we concentrate on quasi-slab modes so that $\hat{g}_{\mathrm{e}, l}\left(k_{r} \Delta_{1}-\theta\right) \cong \hat{g}\left(k_{\mathrm{r}} \Delta_{\mathrm{l}}\right)-\theta \partial \hat{g} / \partial\left(k_{\mathrm{r}} \Delta_{\mathrm{l}}\right)$.

In the conventional theory, magnetic shear leads to damping of the electron slab branch; this has been attributed to energy radiation which is concomitant with the oscillating-outgoing character of the solutions [8]. The conventional theory, however, considers the trapped electron response as a perturbation; this is particularly inadequate for the collisionless electron drift branch where the ratio of the perturbed trapped and passing electron densities is of order unity. Defining the normalized radial wave vector $\hat{k}_{\mathrm{r}}=k_{\mathrm{r}} a_{\mathrm{s}} \sqrt{\left|L_{\mathrm{S}} / L_{\mathrm{N}}\right|}$, the ion-sound Larmor radius $a_{\mathrm{s}}=\sqrt{T_{\mathrm{e}} m_{\mathrm{i}}} / e B$ and the density and magnetic shear lengths $L_{\mathrm{N}}=\partial_{\mathrm{r}} \ln N_{\mathrm{e}}$ and $L_{\mathrm{S}}=q R / \hat{s}$, the corrected quasi-slab eigenvalue equation reads

$$
\left\{\varpi_{l}+\frac{\partial^{2}}{\partial \hat{k}_{\mathrm{r}}^{2}}+\left[1+2 q^{2}\left(1+\mathrm{i} \delta_{\mathrm{i}} \operatorname{sign} q\right)\right] \hat{k}_{\mathrm{r}}^{2}+\operatorname{sign}\left(L_{\mathrm{N}} / L_{\mathrm{S}}\right)\left(-\hat{s}^{-1} \delta_{0}+\delta_{1} \frac{\hat{k}_{\mathrm{r}} \partial}{\partial \hat{k}_{\mathrm{r}}}\right)\right\} \hat{\varphi}_{l}^{(0)}=0 ;
$$

see [7]. Here, $\hat{\varphi}_{l}=-\mathrm{e} \Phi_{l} / T_{\mathrm{e}}$ is the normalized electrostatic potential,

$$
\varpi_{l}=\left[1-<\left(f_{\mathrm{e}}\right)_{\text {trap }}>_{\theta}\right] \frac{\left|L_{\mathrm{S}}\right|}{\left|L_{\mathrm{N}}\right|} \frac{\omega_{l}^{\prime}-\omega_{\mathrm{e}, l}^{*}}{\alpha_{\mathrm{i}} \omega_{\mathrm{e}, l}^{*}},
$$

the eigenvalue $\partial^{2} / \partial \hat{k}_{\mathrm{r}}^{2}$ corresponds to the ion motion parallel to the magnetic field, $\hat{k}_{\mathrm{r}}^{2}$ represents finite Larmor radius effects, $2 q^{2}\left(1+\mathrm{i} \delta_{\text {isign } q}\right) \hat{k}_{\mathrm{r}}^{2}$ arises from the ion dynamics in relation to the side-bands ( $\mathrm{i} \delta_{\mathrm{i}}$ corresponds in particular to ion resonant interaction) and the $\delta_{0}$ and $\delta_{1}$ terms result from the bounce average of $k \cdot v_{B, \text { trap }} \hat{g}_{\mathrm{e}, l}$. Thus

$$
\begin{aligned}
\delta_{0,1}=\frac{3 \sqrt{2 \varepsilon} \eta_{\mathrm{e}} q}{4 \alpha_{\mathrm{i}}} & <\int_{|\theta|}^{\pi} h\left(\theta_{\mathrm{b}}\right) \mathrm{d} \theta_{\mathrm{b}}\left(\int_{-\theta_{\mathrm{b}}}^{\theta_{\mathrm{b}}} \frac{\mathrm{d} \theta^{\prime}}{\sqrt{\cos \theta^{\prime}-\cos \theta_{\mathrm{b}}}}\right)^{-1} \int_{-\theta_{\mathrm{b}}}^{\theta_{\mathrm{b}}} \frac{\left(\cos \theta^{\prime \prime} ; \theta^{\prime \prime} \sin \theta^{\prime \prime}\right) \mathrm{d} \theta^{\prime \prime}}{\sqrt{\cos \theta^{\prime \prime}-\cos \theta_{\mathrm{b}}}}>_{\theta}, \\
\delta_{\mathrm{i}} & =\sqrt{\frac{\pi}{8}} \frac{\tau_{\mathrm{i}} \eta_{\mathrm{i}}}{4 \alpha_{\mathrm{i}}}\left(q R \omega_{\mathrm{e}, l}^{*} / c_{\mathrm{i}}\right)^{5} \exp \left[-\left(q R \omega_{\mathrm{e}, l}^{*}\right)^{2} / 2 c_{\mathrm{i}}^{2}\right],
\end{aligned}
$$

where $\alpha_{\mathrm{i}}=1+\tau_{\mathrm{i}}\left(1+\eta_{\mathrm{i}}\right), \tau_{\mathrm{i}}=T_{\mathrm{i}} / T_{\mathrm{e}}, \quad \eta_{\mathrm{e}, \mathrm{i}}=L_{N} / L_{\mathrm{Te}, \mathrm{i}}, \varepsilon=r / R, h\left(\theta_{\mathrm{b}}\right)=$ $\sin \theta_{\mathrm{b}} / \sqrt{2\left(\cos \theta-\cos \theta_{\mathrm{b}}\right)}$ and the domain $[\theta, \pi]$ includes all trapped electrons at the position $\theta$ (their bouncing angle must indeed be larger than $\theta$ and smaller than $\pi$; we assume $0<\theta<\pi$ for clarity). Finally, $\left(f_{\mathrm{e}}\right)_{\text {trap }}$ is the fraction of trapped electrons, $\omega_{\mathrm{e}, l}^{*}=l T_{\mathrm{e}} \partial_{\mathrm{r}} \ln N_{\mathrm{e}} / e R B_{\theta}$ and $<>_{\theta}$ refers to a poloidal angle average. Noting that $\delta_{1}=\left|\delta_{1}\right| \operatorname{sign} \eta_{\mathrm{e}}, \delta_{\mathrm{i}}=\left|\delta_{\mathrm{i}}\right| \operatorname{sign} \eta_{\mathrm{i}} \omega_{\mathrm{e}, l}^{*}$ and $\left(\delta_{1}\right)^{2}<<4\left(1+2 q^{2}\right)$, the orthogonal eigenfunctions and eigenvalues of equation (1) are

$$
\begin{aligned}
& \hat{\varphi}_{l, n}^{(0)}=\exp \left(-\xi \hat{k}_{\mathrm{r}}^{2} / 2\right) H_{\mathrm{n}}\left[\hat{k}_{\mathrm{r}} \sqrt{\xi-0.5 \delta_{1} \operatorname{sign}\left(L_{\mathrm{N}} / L_{\mathrm{S}}\right)}\right], \\
& \varpi_{l, n}=(2 n+1) \xi+\left(\hat{s}^{-1} \delta_{0}-n \delta_{1}\right) \operatorname{sign}\left(L_{\mathrm{N}} / L_{\mathrm{S}}\right),
\end{aligned}
$$

where the $H_{n}$ s are Hermite polynomials and

$$
\xi \cong \frac{\left|\delta_{1}\right|}{2} \operatorname{sign}\left(\hat{s} L_{\mathrm{Te}}\right) \frac{q^{2}\left|\delta_{\mathrm{i}}\right|}{\sqrt{1+2 q^{2}}} \operatorname{sign}\left(\eta_{\mathrm{i}} \omega_{\mathrm{e}, l}^{*}\right) \pm \mathrm{i} \sqrt{1+2 q^{2}}
$$


Those results lead to the following conclusions:

(i) The growth / damping rate is

$$
\gamma_{l, n}=\Im m \omega_{l}^{\prime(2)}= \pm(2 n+1) \sqrt{1+2 q^{2}} \frac{\left|L_{\mathrm{N}}\right|}{\left|L_{\mathrm{S}}\right|} \frac{\alpha_{i} \omega_{\mathrm{e}, l}^{*}}{1-<\left(f_{\mathrm{e}}\right)_{\text {trap }}>_{\theta}} ;
$$

since the inverse Fourier transform of $(2 a)$ for $n=0$ reads

$$
\begin{aligned}
& \hat{\varphi}_{l, n=0}^{(0)}(\hat{x}, t) \propto \exp \left(-\frac{\hat{x}^{2}}{2 \xi}-\mathrm{i} \omega t\right) \cong \exp \left[-\frac{\hat{x}^{2} \Re \mathrm{e} \xi}{2\left(1+2 q^{2}\right)}+\gamma_{l, n=0} t\right] \\
& \exp \left[ \pm \mathrm{i}\left(\frac{\hat{x}^{2}}{2 \sqrt{1+2 q^{2}}}\right) \mp \omega_{\mathrm{e}, l}^{*} t\right]
\end{aligned}
$$

bounded unstable solutions [corresponding to $\pm \omega_{\mathrm{e}, l}^{*}>0$, according to (3)] propagate away from the rational surface (outgoing disturbances); bounded damped solutions propagate towards that surface;

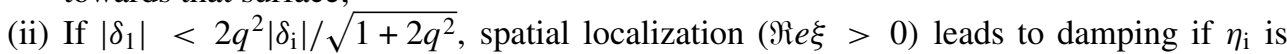
positive and to growth if $\eta_{\mathrm{i}}$ is negative;

(iii) If $\left|\delta_{1}\right|>2 q^{2}\left|\delta_{\mathrm{i}}\right| / \sqrt{1+2 q^{2}}$, spatial localization requests that $\operatorname{sign} L_{\mathrm{Te}}=\operatorname{sign} \hat{s}$, i.e. negative magnetic shear if, as is usually the case, $L_{\mathrm{Te}}$ is negative; here, the trapped electron response leads to a pair of growing and damped solutions; there is no physically meaningful (bounded) solution if $\operatorname{sign} L_{\mathrm{Te}}=-\operatorname{sign} \hat{s}$.

Requiring $\left|q R \omega_{\mathrm{e}, l}^{*} / c_{\mathrm{i}}\right|>>1$ in order to minimize ion Landau damping for $\eta_{\mathrm{i}}>0$, the selfconsistent electrostatic potential $\left(\Phi_{l}^{(1)}\right)_{\mathrm{s}-\mathrm{b}}$ associated with the side-bands reads $\left(\Phi_{l}^{(1)}\right)_{\mathrm{s}-\mathrm{b}}=$ $\left[\hat{\Phi}_{l}^{(1)}\right]_{\sin } \sin \theta$, where

$$
\left[\hat{\Phi}_{l}^{(1)}\right]_{\mathrm{sin}} \cong 2 \frac{q R \omega_{\mathrm{e}, l}^{*}}{c_{\mathrm{i}}} \frac{B_{\varphi}}{B} q k_{\mathrm{r}} a_{\mathrm{i}} \hat{\Phi}_{l}^{(0)}\left(1+2 \mathrm{i} \delta_{\mathrm{i}} \operatorname{sign} q\right) .
$$

The factor $q R \omega_{\mathrm{e}, l}^{*} / c_{\mathrm{i}}$ arises here as the ion $\nabla B$ and curvature drifts are proportional to $c_{\mathrm{i}} / R \omega_{\mathrm{e}, l}^{*}$ and the dispersion function $D_{\mathrm{s}-\mathrm{b}}=D\left(\omega_{\mathrm{e}, l}^{*}, c_{\mathrm{i}} / q R<<\omega_{\mathrm{e}, l}^{*}\right)$ is of the order of $\left(c_{\mathrm{i}} / q R \omega_{\mathrm{e}, l}^{*}\right)^{2}$ (since $D\left(\omega_{\mathrm{e}, l}^{*}, k_{\|} c_{\mathrm{i}} \rightarrow 0\right)=0$ for the primary oscillation). This factor also appears in the first order perturbed density, pressure and $E \times B$ velocity. The expression of the anomalous heat flux obtained from quasi-linear theory is thus boosted by $\left(q R \omega_{\mathrm{e}, l}^{*} / c_{\mathrm{i}}\right)^{2}$

$$
\begin{aligned}
& \frac{Q_{\mathrm{e}}}{P_{\mathrm{e}}}=\frac{2 \alpha_{\mathrm{i}} T_{\mathrm{e}}}{\left|e_{\mathrm{e}} B_{\varphi}\right| r} \frac{-L_{\mathrm{N}}}{R}\left(\frac{q R \omega_{\mathrm{e}, l}^{*}}{c_{\mathrm{s}}}\right)^{2} \frac{<H_{3}+2\left(H_{2}-H_{1}\right)>_{\theta}}{<\Im_{1}>_{\theta}} \sqrt{1+2 q^{2}}|\hat{s}| \\
& \sum_{1, \mathrm{n}}|l|(2 n+1){\overline{\mid \varphi_{1, \mathrm{n}}}}^{2} .
\end{aligned}
$$

This is not the case for the particle flux in view of the relation $\hat{n}_{\mathrm{e}, l}^{(1)} / N_{\mathrm{e}}=\hat{\varphi}_{l}^{(1)}$ which still holds although the perturbed trapped electron distribution function is not a Maxwellian. Thus

$$
\begin{aligned}
& \frac{\Gamma_{\mathrm{e}}}{N_{\mathrm{e}}}=\frac{\alpha_{\mathrm{i}} T_{\mathrm{e}}}{\left|e_{\mathrm{e}} B_{\varphi}\right| r} \frac{-L_{\mathrm{N}}}{R} \frac{\sqrt{1+2 q^{2}}}{1-<\left(f_{\mathrm{e}}\right)_{\text {trap }}>_{\theta}}|\hat{s}| \sum_{1, \mathrm{n}}|l|(2 n+1){\overline{\mid \varphi_{l, n}}}^{2} . \\
& {\overline{\left|\varphi_{l, n}\right|}}^{2}=\left|\Delta_{l}\right|^{-1} \int_{-\infty}^{\infty} \mathrm{d} x\left|\varphi_{1, \mathrm{n}}^{(0)}\left(x=r-r_{l, m}, \mathrm{r}\right)\right|^{2}=\left|\Delta_{l}\right|^{-1} 2 \pi \\
& \int_{-\infty}^{\infty} \mathrm{d} k_{\mathrm{r}}\left|\varphi_{l, n}^{(0)}\left(k_{\mathrm{r}}, r\right)\right|^{2}
\end{aligned}
$$


is the local spatial average of the turbulence level for the nth Hermite polynomial solution and the $H$ s are various integrals over the trapped particle bouncing angle $\theta_{\mathrm{b}}$ :

$$
\begin{aligned}
& H_{1,3}(\theta)=(\cos \theta ; \theta \sin \theta) \int_{\theta}^{\pi} h\left(\theta_{\mathrm{b}}\right) \mathrm{d} \theta_{\mathrm{b}} \\
& H_{2}(\theta)=\int_{\theta}^{\pi}\left[h\left(\theta_{\mathrm{b}}\right)\left(\int_{-\theta_{\mathrm{b}}}^{\theta_{\mathrm{b}}} \frac{\mathrm{d} \theta}{v_{\|}}\right)^{-1}\left(\int_{-\theta_{\mathrm{b}}}^{\theta_{\mathrm{b}}} \frac{\mathrm{d} \theta}{v_{\|}} \cos \theta\right)\right] \mathrm{d} \theta_{\mathrm{b}} .
\end{aligned}
$$

We note that ${\overline{\mid \varphi_{l, n}}}^{2}$ is proportional to the absolute value of the magnetic shear parameter $|\hat{s}|$ as the unstable modes are separated by $\left|\Delta_{l}\right|=1 /\left|l \partial_{\mathrm{r}} q\right|$; this is one of the many reasons why an ITB forms preferentially near flux surfaces where magnetic shear is weak. The unusually large ratio $Q_{\mathrm{e}} / T_{\mathrm{e}} \Gamma_{\mathrm{e}} \approx\left(q R \omega_{\mathrm{e}, l}^{*} / c_{\mathrm{s}}\right)^{2}$ is an important result that has been ignored in the literature where the ballooning limit has mostly been considered.

\section{Application to electron ITBs}

The collisionless reversed magnetic shear trapped electron instability discussed in the previous section may explain the flat electron temperature profile observed in the core of negative central shear discharges such as that described in [1] and [2].

According to [9], trapped electron collisions destabilize the TEM if $\left|\omega_{\mathrm{e}, l}^{*}\right|<3.64 v_{\mathrm{e}, \mathrm{eff}}$ and damp it otherwise $\left(v_{\mathrm{e}, \text { eff }}=v_{\mathrm{e}} / \varepsilon\right.$ is the effective trapped electron collision frequency; a factor $3 \sqrt{3}=5.2$ arises with respect to [9] in view of different definitions). Taking ion Landau damping on the side-bands into account yields a condition on $\omega_{\mathrm{e}, l}^{*}$, namely, $c_{\mathrm{i}} / q R_{0} \omega_{\mathrm{e}, l}^{*}<O\left(1 / \sqrt{5)}\right.$ corresponding to $\partial \delta_{\mathrm{i}} / \partial\left(q R \omega_{\mathrm{e}, l}^{*} / c_{\mathrm{i}}\right)<0$. A barrier could thus develop at the position where

$$
c_{\mathrm{i}} / q R v_{\mathrm{e}, \mathrm{eff}} \cong \frac{2.4 \varepsilon \sqrt{\tau_{\mathrm{i}} / A_{\mathrm{i}}}\left(T_{\mathrm{e}} / 10^{3} e \mathrm{~V}\right)^{2}}{q R(m) Z_{\mathrm{eff}}\left(N_{\mathrm{e}} / 10^{20} \mathrm{~m}^{-3}\right)} \leqslant O(3.64 / \sqrt{5}=1.63),
$$

$A_{\mathrm{i}}$ being the ion atomic mass. It has been shown in [7] that this condition is satisfied at the foot of the barrier pedestal if $Z_{\text {eff }} / \sqrt{\tau_{\mathrm{i}}} \cong 12$. The weakness of the scenario however resides in that the collisionality constraint (8) must be satisfied concomitantly with the condition $\hat{s} \cong 0$.

A more likely scenario is obtained by combining the collisionless negative and positive magnetic shear instabilities discussed here and in [4] and [5]. Thus,

(i) the resonant collisionless instability prevails where $\hat{s}>-0.57 / 0.64=-0.89$ [5], i.e. roughly outside the barrier, while the collisionless reversed magnetic shear instability prevails inside, leading to weak temperature gradients in both regions and

(ii) the growth (or damping) rates of both instabilities are weak within the barrier itself, giving rise to a large local temperature gradient.

The difference between the measured temperature and density profiles can be further understood in the light of the predicted large core value of $Q_{\mathrm{e}} / T_{\mathrm{e}} \Gamma_{\mathrm{e}}$ since continuity of the particle flux through the barrier should not require a local density gradient as steep as the temperature gradient.

Quasi-linear theory predicts neither the turbulence level nor the anomalous transport rate. Moreover, the density and temperature profiles also depend on the distribution of the particle and energy sources. Thus, full justification of the above suggestions clearly requires additional work. 


\section{Summary and comments}

We have shown that a proper extension of the theory of the trapped electron mode which takes into account the effect of the $\nabla B$ and curvature drifts on both the trapped electron and the ion responses can explain the profiles observed in negative central shear high temperature, low density JT-60U and TCV discharges with internal electron transport barriers. In the most likely scenario, the positive magnetic shear instability discussed in [4] and [5] prevails outside the barrier and the negative shear instability discussed in section 2 and [7] prevails inside. Here, structure of the instability drive favours the quasi-slab branch. Quasi-linear theory yields the ratio of the particle and heat fluxes. The latter is enhanced by the presence of the side-bands so that $Q_{\mathrm{e}} / T_{\mathrm{e}} \Gamma_{\mathrm{e}} \gg 1$. This result may explain the marked difference between the measured density and electron temperature profiles. The theory is reactor relevant.

Our results for negative magnetic shear plasmas are not supported by the FULL code, a comprehensive kinetic microinstability code which predicted stability in the core of JT-60U RS plasmas [10]. The reason may be related to the fact that the instability drive for negative magnetic shear is expected to strongly decrease for modes ballooning in the equatorial plane.

The unstable modes discussed in section 2 and [7] do correspond to outgoing solutions propagating away from the rational surfaces; the damped modes propagate towards these surfaces. These results contradict the usual notion of magnetic shear damping. The latter actually rests on the asymptotic spatial decay of the modes being justified by the analytic continuation $\omega_{\mathrm{e}, l}^{*} \rightarrow \omega_{\mathrm{e}, l}^{*}+\mathrm{i} \gamma_{l}$, where $\gamma_{l} \rightarrow 0^{+}$(The slab approximation leads to $\xi= \pm \mathrm{i}$ before considering the above analytic continuation, cf, equation $(2 c)$ ). In equation (1), the new trapped electron term proportional to $\delta_{1}$ yields spatial decay of outgoing solutions; it is larger than the term (not included in the equation) which would follow from the analytic continuation $\omega_{\mathrm{e}, l}^{*} \rightarrow \omega_{\mathrm{e}, l}^{*}+\mathrm{i} \gamma_{l}$.

\section{References}

[1] Ikeda Y et al 2002 Proc. 12th Joint workshop on Electron Cyclotron Emission and Electron Cyclotron Heating (Aix-en-Provence, France Singapore: World Scientific) http://wshop.free.fr/ec12/PAPERS/021-Ikeda.pdf

[2] Sauter O et al 2005 Phys. Rev. Lett. 94105002

[3] Hahm T S et al 2004 Plasma Phys. Control. Fusion 46 A323

[4] Adam J C, Tang W M and Rutherford P H 1976 Phys. Fluids. 19561

[5] Li Jiquan and Kishimoto Y 2002 Plasma Phys. Control. Fusion 44 A479

[6] Rogister A L 1995 Phys. Plasmas 22729

[7] Rogister A L and Singh R 2005 Phys. Plasmas 12112106

[8] Pearlstein L D and Berk H L 1969 Phys. Rev. Lett. 23220

[9] Manheimer W M and Lashmore-Davies C N 1989 MHD and Microinstabilities in Confined Plasmas (Bristol: Institute of Physics Publishing) chapter 18

[10] Rewoldt G et al 2002 Nucle. Fusion 42403 